\title{
ON THE EXISTENCE OF COMPACT METRIC SUBSPACES WITH APPLICATIONS TO THE COMPLEMENTATION OF $c_{0}$
}

\author{
BY \\ WILLIAM H. CHAPMAN AND DANIEL J. RANDTKE
}

\begin{abstract}
A topological space $X$ has property $\sigma-$ CM if for every countable family $F$ of continuous scalar valued functions on $X$ there is a compact metrizable subspace $M$ of $X$ such that $f(X)=f(M)$ for every $f$ in $F$. Every compact metric space, every weakly compact subset of a Banach space and every closed ordinal space has property $\sigma-\mathrm{CM}$. Every continuous image of an arbitrary product of spaces having property $\sigma-\mathrm{CM}$ also has property $\sigma-$ CM. If $X$ has property $\sigma-\mathrm{CM}$, then every copy of $c_{0}$ in $C(X)$ is complemented in $C(X)$. If a locally convex space $E$ belongs to the variety of locally convex spaces generated by the weakly compactly generated Banach spaces, then every copy of $c_{0}$ in $E$ is complemented in $E$.
\end{abstract}

We say that a family $F$ of continuous functions from one topological space $X$ into another depends on a compact metric space if there is a compact metrizable subspace $M$ of $X$ such that $f(M)=f(X)$ for every $f$ in $F$. We say that a topological space $X$ has property $\mathrm{CM}$ if each element of $C(X)$ (the continuous scalar valued functions on $X$ ) depends on a compact metric space. We say that a topological space $X$ has property $\sigma-\mathrm{CM}$ if each countable family in $C(X)$ depends on a compact metric space. These definitions originated from an examination of the proof of $[9$, Lemma 6, p. 60] where it is shown that every copy of $c_{0}$ in $C(X)$ is complemented in $C(X)$ whenever $X$ is a dyadic space.

Clearly any topological space having property $\sigma-\mathrm{CM}$ has property $\mathrm{CM}$; and by $[10$, Problem 1G] any space having property $\mathrm{CM}$ is pseudocompact. By [22, Corollary 23.2] it follows that every Hausdorff continuous image of a topological space having property $\sigma-\mathrm{CM}$ [respectively, $\mathrm{CM}$ ] also has property $\sigma-$ CM [respectively, CM] . Theorem 1.4 below implies that a topological space $X$ has property $\sigma-\mathrm{CM}$ whenever $X$ is the continuous image of an arbitrary product of any one of the following types of spaces:

Presented to the Society, November 8, 1974 under the title On the complementation of $c_{0}$ : received by the editors March $11,1975$.

AMS (MOS) subject classifications (1970). Primary 46A05, 46E15, 54B10, 54E35.

Key words and phrases. Metric space, dyadic space, ordinal space, weakly compact subset of a Banach space, weakly compactly generated Banach space, product space, $\Sigma$-product space, sequential limit point, complemented copy of $c_{0}$. 
(a) Compact metric spaces.

(a') Dyadic spaces.

(a") Compact topological groups.

(b) Weakly compact subsets of Banach spaces.

(b') One point compactifications of locally compact metric spaces.

(c) Closed ordinal spaces.

(c') Ordinal spaces of the form $[0, \alpha)$, where $\alpha$ is not the limit of a countable sequence of strictly smaller ordinals.

The first section of this paper is concerned with property $\sigma-\mathrm{CM}$. Theorem 1.4 implies that every continuous image of an arbitrary product of topological spaces having property $\sigma-\mathrm{CM}$ also has property $\sigma-\mathrm{CM}$. Corollary 1.2 below shows that every completely regular space having property $\sigma-\mathrm{CM}$ is a Baire space. Corollary 1.3 shows that every closed $G_{\delta}$ subset of a completely regular space having property $\sigma-\mathrm{CM}$ also has property $\sigma-\mathrm{CM}$. Proposition 1.7 below implies (as a very special case) that the one point compactification of a disjoint union of compact spaces having property $\sigma-\mathrm{CM}$ also has property $\sigma-\mathrm{CM}$.

The second section is concerned with applications to the complementation of $c_{0}$. In [9, Lemma 6, p. 60] Engelking and PeYczynski showed that every copy of $c_{0}$ in $C(X)$ is conplemented in $C(X)$ whenever $X$ is a dyadic space (the continuous image of an arbitrary product of two point discrete spaces). By [1, Theorem 2, p. 37] it follows that every copy of $c_{0}$ in $C(X)$ is complemented in $C(X)$ whenever $X$ is homeomorphic to a weakly compact subset of a Banach space. Proposition 2.1 below generalizes these known results. Proposition 2.1 shows that every copy of $c_{0}$ in $C(X)$ is complemented in $C(X)$ whenever $X$ has property $\sigma-C M$. The classical result of Sobczyk [20, Theorem 5, p. 942] (see also [11], [14], [17] and [21]) states that every copy of $c_{0}$ in $E$ is complemented in $E$ whenever $E$ is a separable normed space. Recently, Diestel and Lohman [5, Theorem 2, p. 42] extended this result to separable locally convex spaces. By [15, Theorem 2.1, p. 243] it follows that every copy of $c_{0}$ in $E$ is complemented in $E$ whenever $E$ is a weakly compactly generated Banach space. Proposition 2.6 below generalizes these known results. Proposition 2.6 states that every copy of $c_{0}$ in $E$ is complemented in $E$ whenever $E$ belongs to the variety (see [6]) of locally convex spaces generated by the class of weakly compactly generated Banach spaces.

The third section of this paper is concerned with property $\mathrm{CM}$ and the existence of sequential limit points. (A nonisolated point $x$ in a topological space $X$ is a sequential limit point of $X$ if $x$ is the limit of a countable sequence in $X \backslash\{x\}$.) We do not know whether properties $C M$ and $\sigma-C M$ are distinct.

For any unexplained notation or terminology we refer the reader to [10], [19], or [22]. All topological spaces are assumed to be Hausdorff. The symbol 
$c_{0}$ denotes the usual Banach space of zero-convergent scalar valued sequences. If $X$ is a pseudocompact space, then $C(X)$ denotes the usual Banach space of continuous scalar valued functions on $X$. By a copy of $c_{0}$ in a locally convex space $E$ we mean the image of a linear homeomorphism from $c_{0}$ into $E$. A Banach space $E$ is weakly compactly generated if $E$ contains a weakly compact subset whose linear span is dense in $E$. For basic results concerning weakly compactly generated Banach spaces we refer the reader to [1] and [15].

1. Property $\sigma-\mathrm{CM}$. Examples of spaces having property $\sigma-\mathrm{CM}$. We first observe that if a topological space $X$ has the property that the closure of every separable subset of $X$ is compact and metrizable, then $X$ has property $\sigma$ CM. (To see this let $F$ be a countable subset of $C(X)$ and let $D$ be a countable subset of $X$ such that $f(D)$ is dense in $f(X)$ for each $f$ in $F$. If $M=\bar{D}$ is compact and metrizable, then $f(M)=f(X)$ for each $f$ in $M$ and $F$ depends on $M$.) Since every compact metric space, every weakly compact subset of a Banach space (see [15]), every closed ordinal space (use a simple transfinite induction argument), and every ordinal space of the form $[0, \alpha)$, where $\alpha$ is not the limit of a countable sequence of strictly smaller ordinal numbers, has the property that every separable subspace has a compact metrizable closure, it follows that each of these spaces has property $\sigma-C M$. By [15, Proposition 3.2] every one point compactification of a locally compact metric space is homeomorphic to a weakly compact subset of a Banach space and, therefore, has property $\sigma-C M$. Since every dyadic space is the continuous image of a product of compact metric spaces, Theorem 1.4 below implies that every dyadic space has property $\sigma-\mathrm{CM}$. By [16, Proposition 7.6] every compact topological group is dyadic and therefore has property $\sigma-\mathrm{CM}$.

Proposition 1.1. For a topological space $X$ the following are equivalent:

(a) $X$ has property $\sigma-\mathrm{CM}$.

(b) For each sequence $\left\{A_{n}\right\}$ of nonempty cozero sets in $X$, there is a compact metrizable subspace $M$ of $X$ that meets each $A_{n}$.

(c) For each sequence $\left\{Z_{n}\right\}$ of nonempty zero sets in $X$, there is a compact metrizable subspace $M$ of $X$ that meets each $Z_{n}$.

(d) Each countable family of continuous functions from $X$ into the Hilbert cube depends on a compact metrix space.

(e) Each continuous function from $X$ into the Hilbert cube depends on a compact metric space.

(f) For every separable subspace $S$ of $C(X)$ there is a compact metrizable subspace $M$ of $X$ such that for each $x$ in $X$ there is an $m$ in $M$ with $f(x)=f(m)$ for every $f$ in $S$. 
Proof. (a) implies (b), (d) implies (e) and (f) implies (a) are clear.

(b) implies (c). Let $\left\{Z_{n}\right\}$ be a sequence of zero sets in $X$. For each $n$ let $f_{n}$ be a continuous function from $X$ into $[0,1]$ with $Z_{n}=\left\{x: f_{n}(x)=1\right\}$. Let $\left\{r_{k}\right\}$ be an increasing sequence in $[0,1)$ that converges to 1 . For each pair $n, k$ of positive integers let

$$
g_{n, k}=\max \left\{f_{n}, r_{k}\right\}-r_{k} .
$$

By (b) there is a compact metrizable subspace $M$ of $X$ and points $x_{n, k}$ in $M$ such that

$$
\max \left\{f_{n}\left(x_{n, k}\right), r_{k}\right\}-r_{k}=g_{n, k}\left(x_{n, k}\right)>0 .
$$

But then $1 \geqslant f_{n}\left(x_{n, k}\right)>r_{k}$. Since the sequence $\left\{r_{k}\right\}$ converges to 1 , it follows that any adherent point of the sequence $\left\{x_{n, k}: k=1,2, \ldots\right\}$ belongs to both $M$ and $Z_{n}$. So, (c) follows.

(c) implies (d). Let $\left\{f_{n}\right\}$ be a sequence of continuous functions from $X$ into the Hilbert cube $\Pi I_{k}$ where $I_{k}=[0,1]$ for each $k=1,2, \ldots$ For each $k$ let $\mathrm{pr}_{k}$ denote the projection of $H$ onto its $k$ th coordinate $I_{k}$. For each pair $n, k$ of positive integers let $f_{n, k}(x)=\operatorname{pr}_{k}\left(f_{n}(x)\right)$. Let $D$ be a countable subset of $H$ such that $D \cap f_{n}(X)$ is dense in $f_{n}(X)$ for each $n$. For each $d=\left\{d_{k}\right\}$ in $D$ and each $n$ let

$$
Z_{d, n}=\left\{x: f_{n}(x)=d\right\}=\bigcap\left\{\left\{x: f_{n, k}(x)=d_{k}\right\}: k \geqslant 1\right\} .
$$

Since each $Z_{d, n}$ is a zero set, (c) implies that there is a compact metrizable subspace $M$ of $X$ that meets each nonempty $Z_{d, n}$. Therefore, $D \cap f_{n}(X) \subset f_{n}(M) \subset$ $f_{n}(X)$ for every $n$. Since $D \cap f_{n}(X)$ is dense in $f_{n}(X)$, it follows that $f_{n}(M)=$ $f_{n}(X)$ for every $n$. So, (d) follows.

(e) implies (f). Let $S$ be a separable subspace of $C(X)$. Let $\left\{f_{n}\right\}$ be a dense sequence in $S \backslash\{0\}$. Let $f$ be the continuous function from $X$ into the Hilbert cube defined by $f(x)=\left\{\left\|f_{n}\right\|^{-1} f_{n}(x)\right\}$. By (e) there is a compact metrizable subspace $M$ of $X$ such that $f(M)=f(X)$. Fix $x$ in $X$ and choose $m$ in $M$ so that $f(x)=f(m)$. But then $f_{n}(x)=f_{n}(m)$ for every $n$ and a simple density argument implies that $f(x)=f(m)$ for every $f$ in $S$.

REMARK. Let $S$ be a separable subspace of $C(X)$ where $X$ has property $\sigma-\mathrm{CM}$; and pick a compact metrizable subspace $M$ of $X$ so that Proposition 1.1(f) is satisfied. For each $x$ in $X$ let $M_{x}=\{m \in M: f(x)=f(m)$ for every $f$ in $S$ \}. One can show that for particular choices of $S, X$ and $M$ the set-valued function $\varphi$ on $X$ defined by $\varphi(x)=M_{x}$ is neither upper nor lower semicontinuous (see [2, p. 112] for the definition of upper and lower semicontinuous set function). We do not know whether it is possible to choose $M$ in Proposition 1.1(f) so that the set function $\varphi$ is upper (and/or lower) semicontinuous. Nor do we 
know whether it is possible to choose $M$ in Proposition 1.1(f) so that there is a continuous "selection" function $g: X \rightarrow M$ such that $g(x) \in M_{x}$ for every $x$ in $X$. If such a continuous "selection" function exists, then it is easy to see that $S$ would be contained in a linear subspace of $C(X)$ which is isometric to $C(M)$ and which is the range of a contractive projection in $C(X)$. (This last statement should be compared with [15, Theorem 2.1].)

Since every topological space having property $\sigma-\mathrm{CM}$ is pseudocompact, [10, Problem 3D] implies that every normal space having property $\sigma-C M$ is countably compact. For compact spaces property $\sigma-\mathrm{CM}$ is not comparable with sequential compactness. If $X$ denotes an uncountable product of unit intervals, then $X$ has property $\sigma-C M$ but is not sequentially compact. If $X$ denotes $[0,1]$ $\times\{0,1\}$ equipped with the order topology determined by the order defined by $(a, u) \leqslant(b, v)$ if and only if $a<b$ or $a=b$ and $u \leqslant v$, then $X$ is a sequentially compact, compact space which does not have property $\sigma-\mathrm{CM}$.

In the proof of the following corollary we use the fact that, for a completely regular space $X$, condition (b) in Proposition 1.1 is equivalent to the property that for every sequence $\left\{U_{n}\right\}$ of nonempty open subsets of $X$ there is a compact metrizable subspace $M$ of $X$ that meets each $U_{n}$. Recall that a topological space $X$ is a Baire space if the intersection of every countable family of dense open sets in $X$ is dense in $X$.

COROLLARY 1.2. Every completely regular space having property $\sigma-\mathrm{CM}$ is a Baire space.

Proof. Let $\left\{G_{n}\right\}$ be a sequence of dense open subsets of a completely regular space $X$, where $X$ has property $\sigma-\mathrm{CM}$. Fix $x$ in $X$ and let $V$ be a neighborhood of $x$ in $X$. Inductively choose a sequence $\left\{U_{n}\right\}$ of nonempty open sets in $X$ such that

(i) $\bar{U}_{n+1} \subset U_{n} \subset V$, and

(ii) $\bar{U}_{n} \subset G_{1} \cap \cdots \cap G_{n}$.

Since $X$ has property $\sigma-\mathrm{CM}$ there is a compact metrizable subspace $M$ of $X$ that meets each $U_{n}$. By (i) there is a point $m \in M \cap\left(\cap \bar{U}_{n}\right) \subset V$. By (ii) $m \in \bigcap G_{n}$. Therefore, every neighborhood of $x$ in $X$ meets $\bigcap G_{n}$ and $X$ is a Baire space.

We note that by modifying the proof of the above corollary one can actualiy show that every completely regular space having property $\sigma-\mathrm{CM}$ is strongly $\alpha$ favorable in the sense of $[2$, p. 117].

COROLLARY 1.3. (a) Every zero set in a topological space having property $\sigma-\mathrm{CM}$ has property $\sigma-\mathrm{CM}$.

(b) Every closed $G_{\delta}$ subset of a completely regular space having property $\sigma-\mathrm{CM}$ has property $\sigma-\mathrm{CM}$. 
Proof. (a) follows from condition (b) of Proposition 1.1.

(b) Let $G$ be a closed $G_{\delta}$ subset of a completely regular space $X$, where $X$ has property $\sigma-\mathrm{CM}$. Let $\left\{U_{n}\right\}$ be a sequence of open sets in $X$ such that $G=$ $\cap U_{n}$. Let $\left\{V_{k}\right\}$ be a sequence of open sets in $X$ such that $G \cap V_{k}$ is nonempty for each $k$. Since $X$ has property $\sigma-\mathrm{CM}$ there is a compact metrizable subset $M$ of $X$ such that $M$ meets $U_{1} \cap \cdots \cap U_{n} \cap V_{k}$ for each pair $n, k$ of positive integers. But then $G \cap M$ is a compact metrizable subset of $G$ that meets each $G \cap V_{k}=\bigcap_{n}\left(U_{1} \cap \cdots \cap U_{n} \cap V_{k}\right)$. Therefore, $G$ has property $\sigma-\mathrm{CM}$.

DEFInItion. Let $\left\{X_{\nu}: \nu \in I\right\}$ be a family of topological spaces. Following Corson $\left[4\right.$, p. 786] we say that a subspace $X$ of $\Pi X_{\nu}$ is a $\Sigma$-product of the $X_{\nu}$ if there is a point $p$ in $\Pi X_{\nu}$ such that $x \in X$ if and only if $x_{\nu} \neq p_{\nu}$ for at most a countable number of $\nu$ in $I$. Such a point $p$ is called a base point of this $\Sigma$-product. Corson $[4$, p. 786$]$ has noted that different $\Sigma$-products of the same $X_{\nu}$ are not necessarily homeomorphic.

THEOREM 1.4. If $\left\{X_{\nu}: \nu \in I\right\}$ is a family of topological spaces each of which has property $\sigma-\mathrm{CM}$, then every $\Sigma$-product of the $X_{\nu}$ has property $\sigma-\mathrm{CM}$.

Proof. By adjoining an infinite number of one point discrete spaces to the family $\left\{X_{\nu}: \nu \in I\right\}$, we may assume that $I$ is infinite.

Let $X$ be a $\Sigma$-product of the $X_{\nu}$ with base point $p$. Let $\left\{f_{n}\right\}$ be a sequence of nonzero functions in $C(X)$ and for each $n$ let $A_{n}$ denote the cozero set of $f_{n}$. Let $D$ be a countable subset of $X$ that meets each $A_{n}$. Since $X$ is a $\Sigma$-product of the $X_{\nu}$ with base point $p$, there is a countably infinite subset $J$ of $I$ such that $d_{\nu}$ $=p_{\nu}$ whenever $d \in D$ and $\nu \in J$. To complete the proof it suffices to show that $\Pi\left\{X_{\nu}: \nu \in J\right\}$ has property $\sigma-\mathrm{CM}$. (To see this let $\varphi: \Pi\left\{X_{\nu}: \nu \in J\right\} \rightarrow X$ be defined by $\varphi(x)=y$, where $y_{\nu}=x_{\nu}$ if $\nu \in J$ and $y_{\nu}=p_{\nu}$ if $\nu \notin J$. If $\Pi\left\{X_{\nu}\right.$ : $\nu \in J\}$ has property $\sigma-\mathrm{CM}$, then there is a compact metrizable subspace $M$ of $\Pi\left\{X_{\nu}: \nu \in J\right\}$ such that $M$ meets each of the cozero sets $\varphi^{-1}\left(A_{n}\right)$. By [22, 23.2, p. 166] $\varphi(M)$ is a compact metrizable subset of $X$. Since $M$ meets each $\varphi^{-1}\left(A_{n}\right)$, $\varphi(M)$ meets each $A_{n}$ and by Proposition $1.1 X$ has property $\sigma-C M$.) Therefore without loss of generality we assume that $J=I$ is the set of positive integers.

For every $n$ in $I, d$ in $D$ and $x$ in $X_{1}$ let

$$
g_{n, d}(x)=f_{n}\left(x, d_{2}, d_{3}, \ldots\right) .
$$

Since $\left\{g_{n, d}\right\}$ is a countable family of continuous functions on $X_{1}$, there is a compact metric subspace $M_{1}$ of $X_{1}$ such that every $g_{n, d}\left(M_{1}\right)=g_{n, d}\left(X_{1}\right)$. Let $N_{1}$ be a countable subset of $M_{1}$ such that $f_{n}(d) \in g_{n, d}\left(N_{1}\right)$ for every $n \in I$ and $d \in D$. Suppose that we have found sets $M_{1}, \ldots, M_{k}$ and $N_{1}, \ldots, N_{k}$ so that

(a) $N_{t} \subset M_{t} \subset X_{t}, N_{t}$ is countable and $M_{t}$ is compact and metrizable; and 
(b) for every $\left(n_{1}, \ldots, n_{k-1}\right) \in N_{1} \times \cdots \times N_{k-1}, d \in D$, and $n \in I$, there is an $n_{k} \in N_{k}$ such that

$$
f_{n}(d)=f_{n}\left(n_{1}, \ldots, n_{k} d_{k+1}, \ldots\right) .
$$

For every $q=\left(n_{1}, \ldots, n_{k}\right) \in N_{1} \times \cdots \times N_{k}, d \in D, n \in I$ and $x \in$ $X_{k+1}$ let

$$
g_{q, d, n}(x)=f_{n}\left(n_{1}, \ldots, n_{k}, x, d_{k+2}, \ldots\right) .
$$

Since $\left\{g_{q, d, n}\right\}$ is a countable family of continuous functions on $X_{k+1}$, there is a compact metrizable subspace $M_{k+1}$ of $X_{k+1}$ such that every $g_{q, d, n}\left(M_{k+1}\right)=$ $g_{q, d, n}\left(X_{k+1}\right)$; and there is a countable subset $N_{k+1}$ of $M_{k+1}$ such that

$$
f_{n}\left(n_{1}, \ldots, n_{k}, d_{k+1}, d_{k+2}, \ldots\right) \in g_{q, d, n}\left(N_{k+1}\right)
$$

for every $q=\left(n_{1}, \ldots, n_{k}\right) \in N_{1} \times \cdots \times N_{k}, d \in D$, and $n \in I$. Therefore (by induction), there exist sequences $\left\{M_{k}\right\}$ and $\left\{N_{k}\right\}$ such that (a) and (b) hold for every positive integer $k$.

Let $M=\Pi M_{k}$. By $[22,22.3$, p. 161] $M$ is a compact metrizable subspace of $X$. Fix $n$ in $I$ and let $d \in D$. By (b) there is a point $q=\left(n_{1}, n_{2}, \ldots\right)$ in $\Pi N_{k}$ such that for each $k$

$$
f_{n}(d)=f_{n}\left(n_{1}, \ldots, n_{k}, d_{k+1}, d_{k+2}, \ldots\right) .
$$

Since $q=\lim _{k \rightarrow \infty}\left(n_{1}, \ldots, n_{k}, d_{k+1}, d_{k+2}, \ldots\right)$, it follows that $f_{n}(d)=f_{n}(q)$. Therefore, $f_{n}(D) \subset f_{n}(M)$. Since $D$ meets each $A_{n}=\left\{x: f_{n}(x) \neq 0\right\}$, it follows that $M$ meets each $A_{n}$. By Proposition $1.1 X$ has property $\sigma-\mathrm{CM}$.

COROLlaRY 1.5. Let $\left\{X_{\nu}: \nu \in I\right\}$ be a family of topological spaces each of which has property $\sigma-\mathrm{CM}$. If $\Sigma \subset X \subset \Pi X_{\nu}$ where $\Sigma$ is a $\Sigma$-product of the $X_{\nu}$, then $X$ (and every continuous image of $X$ ) has property $\sigma-\mathrm{CM}$.

Proof. This is an easy consequence of Theorem 1.4 and the fact that every $\Sigma$-product of the $X_{\nu}$ is dense in $\Pi X_{\nu}$.

COROLlaRY 1.6. (a) If $\left\{\alpha_{\nu}\right\}$ is a collection of ordinal numbers, then $\Pi\left[0, \alpha_{\nu}\right)$ has property $\sigma-\mathrm{CM}$ if and only if no $\alpha_{\nu}$ is the limit of a countable sequence of strictly smaller ordinals.

(b) There exists nonnormal spaces having property $\sigma-\mathrm{CM}$.

Proof. (a) follows from Proposition 1.1 and the fact that $[0, \alpha)$ is not pseudocompact (and hence not $\sigma-\mathrm{CM}$ ) whenever $\alpha$ is the limit of a countable sequence of strictly smaller ordinals. 
(b) In [3, Theorem 3, p. 223] Conover has characterized the normality of product spaces of the form $\Pi\left[0, \alpha_{\nu}\right)$. When Conover's characterization is compared with part (a) above, (b) follows.

Definition. Let $X$ be a topological space and let $I$ denote the isolated points of $X$. Let $\left\{X_{\nu}: \nu \in I\right\}$ be a family of topological spaces and let $Y$ denote the set-theoretic disjoint union of the sets $X \backslash I, X_{\nu}(\nu \in I)$. For simplicity, we always assume that the sets $X \backslash I, X_{\nu}(\nu \in I)$ are pairwise disjoint. Define a topology on $Y$ as follows:

(a) if $x \in X_{\nu}$, then the filter of neighborhoods of $x$ in $X_{\nu}$ is a filter base for the filter of neighborhoods of $x$ in $Y$; and

(b) if $x \in X \backslash I$, then a filter base of neighborhoods of $x$ in $Y$ is given by sets of the form $[V \cap(X \backslash I)] \cup\left[\cup\left\{X_{\nu}: \nu \in V\right\}\right]$ where $V$ is a neighborhood of $x$ in $X$. The space $Y$ equipped with the topology defined above is called the space obtained from $X$ by attaching the spaces $\left\{X_{\nu}: \nu \in I\right\}$ to the isolated points $I$ of $X$.

For example, if $I$ is a discrete space and $I^{\infty}$ denotes its one point compactification, and if $\left\{X_{\nu}: \nu \in I\right\}$ is a family of compact spaces, then the one point compactification of the topological disjoint union of the spaces $\left\{X_{\nu}: \nu \in I\right\}$ is homeomorphic to the space obtained from $I^{\infty}$ by attaching the spaces $\left\{X_{\nu}: \nu \in I\right\}$ to the isolated points $I$ of $I^{\infty}$. Another large class of examples is obtained by attaching topological spaces to the isolated points of the ordinal spaces.

Proposition 1.7. Let I denote the set of isolated points of a topological space $X$; and let $Y$ be the space obtained from $X$ by attaching a family $\left\{X_{\nu}: \nu \in\right.$ $I\}$ of topological spaces to the isolated points of $X$.

(a) If $X$ is metrizable and each $X_{\nu}$ is metrizable, then $Y$ is metrizable.

(b) If $X$ is compact and each $X_{\nu}$ is compact, then $Y$ is compact.

(c) If $X$ has property $\sigma-\mathrm{CM}$ and each $X_{\nu}$ has property $\sigma-\mathrm{CM}$, then $Y$ has property $\sigma-\mathrm{CM}$.

PROOF. The proofs of (a) and (b) are straightforward and will be left to the reader.

(c) Let $\left\{f_{n}\right\}$ be a sequence of continuous functions on $Y$. Let $D$ be a countable subset of $Y$ such that $f_{n}(D)$ is dense in $f_{n}(Y)$ for every $n$. For each $\nu$ in $I$ fix a point $x_{\nu}$ in $X_{\nu}$. Let $J$ denote the set of all $\nu$ in $I$ such that $D \cap X_{\nu}$ is nonempty. Since $D$ is countable, $J$ is countable. For every positive integer $n, \nu$ in $J$ and $d$ in $D$ let $f_{n, v, d}$ denote the function on $X$ defined by:

$$
\begin{array}{ll}
f_{n, \nu, d}(x)=f_{n}(x) & \text { if } x \in X \backslash I, \\
f_{n, \nu, d}(\mu)=f_{n}\left(x_{\mu}\right) & \text { if } \mu \in I \backslash\{\nu\},
\end{array}
$$




$$
\begin{array}{ll}
f_{n, \nu, d}(\nu)=f_{n}(d) & \text { if } d \in X_{\nu}, \\
f_{n, \nu, d}(\nu)=f_{n}\left(x_{\nu}\right) & \text { if } d \notin X_{\nu} .
\end{array}
$$

Since $\left\{f_{n, \nu, d}\right\}$ is a countable family of continuous functions on $X$, there is a compact metric subspace $M^{*}$ of $X$ such that the family $\left\{f_{n, \nu, d}\right\}$ depends on $M^{*}$. For each $\nu$ in $I \cap M^{*}$ let $M_{\nu}$ be a compact metrizable subspace of $X_{\nu}$ such that $f_{n}\left(M_{\nu}\right)$ $=f_{n}\left(X_{\nu}\right)$ for every $n$. Let

$$
M=\left(M^{*} \backslash I\right) \cup\left(\bigcup\left\{M_{\nu}: \nu \in M^{*} \cap I\right\}\right) .
$$

It is easy to see that $M$ is a space obtained from $M^{*}$ by attaching certain compact metric spaces to the isolated points of $M^{*}$. Therefore, by parts (a) and (b) of this proposition, $M$ is a compact metrizable subspace of $Y$. To complete the proof it suffices to show that $f_{n}(D) \subset f_{n}(M)$ for every $n$.

Let $n$ be a fixed positive integer and let $d$ be a fixed element of $D$. First, suppose that $d \in X \backslash I$. In this case $f_{n}(d)=f_{n, \nu, d}(d)$ for every $\nu$ in $J$. Let $\nu$ be a fixed element of $J$. Since $f_{n, \nu, d}$ depends on $M^{*}$, there is an $m$ in $M^{*}$ such that $f_{n}(d)=f_{n, \nu, d}(m)$. If $m \in X \backslash I$, then $m \in M^{*} \backslash I \subset M$ and

$$
f_{n}(d)=f_{n, \nu, d}(m)=f_{n}(m) \in f_{n}(M) .
$$

If $m \in I$, then (since $d \notin X_{m}$ )

$$
f_{n}(d)=f_{n, \nu, d}(m)=f_{n}\left(x_{m}\right) \in f_{n}\left(M_{n}\right) \subset f_{n}(M) .
$$

Secondly, suppose that $d \in X_{\nu}$ for some fixed $\nu$ in $J$. In this case $f_{n}(d)=$ $f_{n, \nu, d}(v)$. Since $f_{n, \nu, d}$ depends on $M^{*}$ there is an $m$ in $M^{*}$ such that $f_{n}(d)=$ $f_{n, v, d}(m)$. If $m \in X \backslash I$, then $m \in M^{*} \backslash I \subset M$ and $f_{n}(d)=f_{n, v, d}(m)=f_{n}(m) \in$ $f_{n}(M)$. If $m \in I \backslash\{\nu\}$, then

$$
f_{n}(d)=f_{n, \nu, d}(m)=f_{n}\left(x_{m}\right) \in f_{m}\left(M_{m}\right) \subset f_{n}(M) .
$$

If $m=\nu$, then

$$
f_{n}(d) \in f_{n}\left(X_{m}\right)=f_{n}\left(M_{m}\right) \subset f_{n}(M) .
$$

2. The complementation of $c_{0}$.

Proposition 2.1. If a topological space $X$ has property $\sigma-\mathrm{CM}$, then every copy of $c_{0}$ in $C(X)$ is complemented in $C(X)$.

Moreover, if $T: c_{0} \rightarrow C(X)$ is a linear homeomorphism from $c_{0}$ into $C(X)$ where $X$ has property $\sigma-\mathrm{CM}$, then there is a projection $p$ from $C(X)$ onto $T\left(c_{0}\right)=F$ such that $\|p\| \leqslant 2\|T\|\left\|T^{-1}\right\|$. 
Proof. Let $T: c_{0} \rightarrow C(X)$ be a linear homeomorphism (into) and let $F=$ $T\left(c_{0}\right)$. Let $D$ be a countable dense subset of $F$. Since $X$ has property $\sigma-C M$, $D$ depends on a compact metric space $M$. Since the set of all $f$ in $C(X)$ such that $f(M)=f(X)$ is closed in $C(X)$, it follows that $F$ depends on $M$. Let $R: C(X)$ $\rightarrow C(M)$ denote the restriction operators. Since $F$ depends on $M, R \mid F$ is an isometry. By Sobczyk's theorem [20] there is a continuous linear operator $Q$ from $C(M)$ onto $R T\left(c_{0}\right)$ such that $\|Q\| \leqslant 2\|R T\|\left\|(R T)^{-1}\right\| \leqslant 2\|T\|\left\|T^{-1}\right\|$. Let $P=(R \mid F)^{-1} Q R$; then $P$ is a continuous linear projection from $C(X)$ onto $F=$ $T\left(c_{0}\right)$ such that $\|P\| \leqslant 2\|T\|\left\|T^{-1}\right\|$.

By mimicking the "function space method" proof of Theorems 3,4 and 5 in $[9$, p. 61], one can prove the following generalizations of these theorems.

COROLlARY 2.2. (a) (CF. [9, THEOREM 5]). If a topological space $X$ has property $\sigma-\mathrm{CM}$, then there is no linearly homeomorphic copy of $l_{\infty}$ in $C(X)$.

(b) (CF. [9, THEOREM 3]). If $X$ is a completely regular space such that $\beta(X)$ (the Stone-Čech compactification of $X$ ) has property $\sigma-\mathrm{CM}$, then $X$ is pseudocompact.

(c) (CF. [9, THEOREM 4]). There is no infinite extremally disconnected space having property $\sigma-\mathrm{CM}$.

COROLLARY 2.3. (a) The one point compactification of a locally compact metric space always has property $\sigma-\mathrm{CM}$.

(b) The Stone-Čech compactification of a noncompact locally compact space never has property $\sigma-\mathrm{CM}$.

Proof. (a) follows from the fact (see [15, Proposition 3.2, p. 247]) that the one point compactification of a locally compact metric space is homeomorphic to a weakly compact subset of a Banach space.

(b) follows from Corollary 2.2 and the fact that a noncompact locally compact space is never pseudocompact.

Definition. We say that a locally convex space $E$ is $\sigma-\mathrm{CM}$ generated if $E$ is generated by a subset $X$ such that $X$ equipped with the weak topology has property $\sigma-\mathrm{CM}$. ( $E$ is generated by $X$ if $\operatorname{sp}(X)$, the linear subspace of $E$ spanned by $X$, is dense in $E$.)

Proposition 1.4 implies that every contimuous linear image of an arbitrary product of weakly compactly generated Banach spaces is $\sigma-\mathrm{CM}$ generated. We note that every separable Banach space and every reflexive Banach space is weakly compactly generated.

LEMMA 2.4. If $E$ is a $\sigma-\mathrm{CM}$ generated locally convex space, then every $\sigma\left(E^{\prime}, E\right)$-compact separable subset of $E^{\prime}$ is $\sigma\left(E^{\prime}, E\right)$-metrizable.

Proof. Let $A$ be a $\sigma\left(E^{\prime}, E\right)$-compact separable subset of $E^{\prime}$. Let $X$ be a 
subset of $E$ such that $X$ generates $E$ and $X$ equipped with the $\sigma\left(E^{\prime}, E\right)$-topology has property $\sigma-\mathrm{CM}$. Since $A$ is separable it is easy to see that there is a compact metrizable subspace $M$ of $X$ such that $a(M)=a(X)$ for every $a$ in $\operatorname{sp}(A)$. Let $\left\{x_{n}\right\}$ be a dense sequence in $M$ and for each positive integer $n$ and each $a$ in $E^{\prime}$ let $\rho_{n}(a)=\left|\left\langle x_{n}, a\right\rangle\right|$. The topology $T$ on $E^{\prime}$ generated by the seminorms $\left\{\rho_{n}\right\}$ is weaker than $\sigma\left(E^{\prime}, E\right)$. Since $a(M)=a(X)$ for every $a$ in $\operatorname{sp}(A)$ and since $\left\{x_{n}\right\}$ is dense in $M$, it is easy to see that the restriction of $T$ to $\operatorname{sp}(A)$ is metrizable. Since $T \subset \sigma\left(E^{\prime}, E\right)$ and $A$ is $\sigma\left(E^{\prime}, E\right)$-compact, the topologies $T$ and $\sigma\left(E^{\prime}, E\right)$ coincide on $A$. Therefore, $A$ is $\sigma\left(E^{\prime}, E\right)$-metrizable.

Proposition 2.5. If $E$ is a $\sigma-\mathrm{CM}$ locally convex space, then every copy of $c_{0}$ in $E$ is complemented in $E$.

Proof. Let $T: c_{0} \rightarrow E$ be a linear homeomorphism from $c_{0}$ onto $F=$ $T\left(c_{0}\right)$ and let $U$ be a closed balanced convex neighborhood of 0 in $E$ such that

(i) $U \cap F \subset\{T x:\|x\| \leqslant 1\}$.

Let $\left\{x_{n}\right\}$ be a dense sequence in $c_{0}$ and let $D$ be a countable subset of $U^{0}$ such that for every $n$

(ii) $\left\{\left\langle T x_{n}, a\right\rangle: a \in D\right\}$ is dense in $\left\{\left\langle T x_{n}, a\right\rangle: a \in U^{0}\right\}$. Let $M$ denote the $\sigma\left(E^{\prime}, E\right)$-closure of $D$ in $E$ equipped with the $\sigma\left(E^{\prime}, E\right)$-topology. By Lemma 2.4 $M$ is compact and metrizable. Let $S: E \rightarrow C(M)$ be the linear operator defined by $(S x)(a)=\langle x, a\rangle$ for every $x$ in $E$ and $a$ in $M$. Since $M \subset U^{0}, S$ is continuous. By (ii) above,

$$
\left\|S T x_{n}\right\|=\sup \left\{\left|\left\langle T x_{n}, a\right\rangle\right| a \in U^{0}\right\}
$$

for every $n$. Since $\left\{x_{n}\right\}$ is dense in $c_{0}$ (iii) implies that

$$
\|S T x\|=\sup \left\{|\langle T x, a\rangle|: a \in U^{0}\right\}
$$

for every $x \in c_{0}$. If $\|S T x\| \leqslant 1$, then (by (iv)) $T x \in U$ and (by (i)) $\|x\| \leqslant 1$. Therefore,

$$
\{T x:\|S T x\| \leqslant 1\} \subset\{T x:\|x\| \leqslant 1\}
$$

and it follows that $S \mid F$ is a homeomorphism. By Sobczyk's theorem [22] there is a continuous linear projection $Q$ from $C(M)$ onto $S(F)$. Let $P=(S \mid F)^{-1} Q S$. It is easy to see that $P$ is a continuous linear projection from $E$ onto $F=T\left(c_{0}\right)$.

In [6] Diestel, Morris and Saxon define a variety (of locally convex spaces) to be a nonempty class of locally convex spaces closed under the operations of taking subspaces, quotient spaces (modulo closed subspaces), arbitrary cartesian products and isomorphic images. The variety generated by a class of locally convex spaces is defined to be the smallest variety containing the class. By using [19, 5.4, p. 53] one can show that every separable locally convex space belongs to the 
variety generated by the weakly compactly generated Banach spaces. By combining [6, Corollary 2.1, p. 209] with Proposition 2.2 it follows that:

Proposition 2.6. If $E$ belongs to the variety generated by the class of weakly compactly generated Banach spaces, then every copy of $c_{0}$ in $E$ is complemented in $E$.

Remarks. (a) By a theorem of James [13, Theorem 4, p. 299] it follows that a subset $X$ of a complete locally convex space is weakly relatively compact whenever $X$ equipped with the weak topology has property $\sigma-\mathrm{CM}$. The converse, however, does not hold. For example, $\beta N$ (the Stone-Čech compactification of the positive integers) is homeomorphic to a weakly compact subset of a complete locally convex space; however, by [10, Problem 60(6), p. 97] $\beta N$ does not have property $\sigma-\mathrm{CM}$.

(b) If $E$ is a $\sigma-\mathrm{CM}$ generated locally convex space, then by combining $[19,5.4$, p. 53] with remark (a) above one can show that $E$ is linearly homeomorphic to a linear subspace of a product of weakly compactly generated Banach spaces. Therefore, it follows that the variety generated by the $\sigma-\mathrm{CM}$ generated locally convex spaces coincides with the variety generated by the weakly compactly generated Banach spaces.

(c) Since there exist compact spaces $X$ having property $\sigma-\mathrm{CM}$ such that $X$ is not homeomorphic to a weakly compact subset of a Banach space (e.g. any uncountable closed ordinal space) [1, Theorem 2, p. 37] implies that there exist compact spaces $X$ having property $\sigma-\mathrm{CM}$ such that $C(X)$ is not weakly compactly generated. However, Lemma 2.4 can be used to show that a compact space $X$ has property $\sigma-C M$ whenever $C(X)$ is $\sigma-\mathrm{CM}$ generated. We do not know whether $C(X)$ belongs to the variety generated by the weakly compactly generated Banach spaces, whenever $X$ is a compact space having property $\sigma-\mathrm{CM}$.

(d) J. Diestel has pointed out that the converse of Proposition 2.6 is false. He noted that $l_{1}(I)$ ( $I$ uncountable) does not belong to the variety generated by the weakly compactly generated Banach spaces, but that every copy of $c_{0}$ in $l_{1}(I)$ is complemented.

(e) We do not know whether property $\sigma-C M$ characterizes those compact Hausdorff spaces for which every copy of $c_{0}$ in $C(X)$ is complemented in $C(X)$. We believe that it does not, but have no counterexample.

3. Property $\mathrm{CM}$ and the existence of sequential limit points. The remainder of the paper is concerned with the existence of sequential limit points and is motivated by $[7$, Corollary 9, p. 257] where it is shown that every nonisolated point of a dyadic space is a sequential limit point. (Proposition 3.4 below generalizes this result.) By Proposition 1.1 it follows that if $X$ is a completely regular space having property $\sigma-\mathrm{CM}$, then (a) the closure of each countable family of 
isolated points in $X$ is metrizable and (b) the set of all sequential limit points in $X$ is dense in the set of nonisolated points of $X$. Propositions 3.1 and 3.2 below show that these results also hold if $X$ has property CM.

Since there exist separable nonmetrizable dyadic spaces, one would not expect that the closure of every countable subset of a space having property $\sigma-$ CM (or CM) would be metrizable. Since the Stone-Čech compactification of the integers is nonmetrizable, one would not, in general, expect that the closure of a countable family of isolated points would be metrizable. Since the one point compactification of any discrete space has property $\sigma-\mathrm{CM}$, one would not expect that the closure of the set of isolated points in a space having property $\sigma-$ CM (or CM) would be metrizable. Finally, since there exist nonisolated points in compact ordinal spaces that are not sequential limit points, one would not expect that every nonisolated point in a space having property $\sigma-\mathrm{CM}$ (or $\mathrm{CM}$ ) would be the limit of a nontrivial convergent sequence.

Proposition 3.1. If a topological space $X$ has property $\mathrm{CM}$, then the closure of each countable set of isolated points in $X$ is metrizable.

Proof. Let $\left\{x_{n}\right\}$ be a sequence of isolated points in $X$. Let $f\left(x_{n}\right)=n^{-1}$ for $n=1,2, \ldots$ and let $f(x)=0$ for $x \neq x_{n}, n=1,2, \ldots f$ is then a continuous function on $X$. Since $X$ has property $\sigma-\mathrm{CM}$, there is a compact metric subspace $M$ of $X$ such that $f(M)=f(X)$. But then $\left\{x_{n}\right\}$ is contained in $M$ and the closure of $\left\{x_{n}\right\}$ is metrizable.

Proposition 3.2. If a completely regular Hausdorff space $X$ has property $\mathrm{CM}$, then the set of sequential limit points of $X$ is dense in the set of all nonisolated points of $X$.

Proof. Suppose that the set of sequential limit points in $X$ is not dense in the set of all nonisolated points of $X$; then $X$ contains an open set $U$ such that:

(a) $U$ contains a nonisolated point $p$ of $X$, and

(b) there are no sequential limit points in $\bar{U}$.

Let $V$ be an open neighborhood of the nonisolated point $p$ such that $\bar{V}$ is properly contained in $U$. Let $\left\{U_{n}\right\}$ be a sequence of nonempty pairwise disjoint open subsets of $V$; and for each $n$ choose a point $x_{n}$ in $U_{n}$ and a continuous function $f_{n}$ from $X$ into $[0,1]$ so that $f_{n}\left(x_{n}\right)=1$ and supp $f_{n} \subset U_{n}$. Let $f=$ $\Sigma 2^{-n} f_{n} . f$ is then a continuous function on $X$ such that $f(X)$ is infinite. Let $M$ be a compact metric subspace of $X$ such that $f(M)=f(X)$. Since supp $f \subset$ $\overline{U U_{n}} \subset \bar{V} \subset U$, where $\bar{V} \neq U$, we may assume that $M \subset \bar{U}$. (We need $\bar{V} \neq U$ in the event that $\bar{V}=\operatorname{supp} f=\{x: f(x)>0\}$ is both open and closed in $X$.) Since $\bar{U}$ contains no sequential limit points, $M$ must be finite. But this contradicts the fact that $f(X)=f(M)$ is infinite. 
LEMMA 3.3. Let $f$ be a continuous closed surjection from one topological space $Y$ onto another $X$ and let $X$ be an element of $X$. The following are equivalent:

(a) The point $x$ is not isolated.

(b) There is a point $y$ in $f^{-1}(x)$ such that $f(V \cap D) \backslash\{x\}$ is nonempty, whenever $V$ is a neighborhood of $y$ in $Y$ and $D$ is dense in $Y$.

(c) There is a point $y$ in $f^{-1}(x)$ such that $(V \cap D) \backslash f^{-1}(x)$ is nonempty, whenever $V$ is a neighborhood of $y$ in $Y$ and $D$ is dense in $Y$.

Proof. (a) implies (b). Suppose that for each $y$ in $f^{-1}(x)$ there is an open neighborhood $V_{y}$ and a dense subset $D_{y}$ of $Y$ such that $f\left(V_{y} \cap D_{y}\right) \subset\{x\}$. Since

$$
\{x\} \subset f\left(V_{y}\right) \subset f \overline{\left(V_{y} \cap D_{y}\right)} \subset \overline{f\left(V_{y} \cap D_{y}\right)} \subset\{x\},
$$

it follows that $f\left(V_{y}\right)=\{x\}$. Therefore, $f^{-1}(x)=\bigcup\left\{V_{y}: y \in f^{-1}(x)\right\}$ is open in $Y$. Since $f$ is closed and surjective $f\left[Y \backslash f^{-1}(x)\right]=X \backslash\{x\}$ is closed in $X$, contradicting the fact that $\{x\}$ is nonisolated.

It is easy to see that (b) implies (a) and that (b) is equivalent to (c).

Recall that a topological space $X$ is first countable if each point of $X$ has a countable neighborhood base.

Proposition 3.4. Let $\left\{X_{\nu}: \nu \in I\right\}$ be a family of first countable spaces.

If $f$ is a continuous closed surjection from $\Pi X_{\nu}$ onto a topological space $X$, then every nonisolated point of $X$ is a sequential limit point in $X$.

PRoof. Let $x \in X$. By Lemma 3.3 there is a point $y=\left\{y_{\nu}\right\}$ in $\Pi X_{\nu}$ such that

(*) $(V \cap D) \backslash f^{-1}(x)$ is nonempty, whenever $V$ is a neighborhood of $y$ in $\Pi X_{\nu}$ and $D$ is dense in $\Pi X_{\nu}$. For each $\nu$ in $I$ let $\left\{V_{\nu, n}: n=1,2, \ldots\right\}$ be a countable nested neighborhood base for $y_{\nu}$ in $X_{\nu}$. For each finite subset $J$ of $I$ let

$$
V(n, J)=\left\{z \in \Pi X_{\nu}: z_{\nu} \in V_{\nu, n} \text { for every } \nu \in J\right\} .
$$

The family $\{V(n, J)\}$ forms a neighborhood base for $y$ in $\Pi X_{\nu}$. Let $D$ denote the dense subspace of $\Pi X_{\nu}$ consisting of all points $z$ such that $z_{\nu}=y_{\nu}$ except possibly for a finite number of $\nu$ 's; and for each $z$ in $D$ let $J(z)=\left\{\nu \in I: z_{\nu} \neq\right.$ $\left.y_{\nu}\right\}$. By using $(*)$ and a simple inductive argument we can show that there is a sequence $\left\{z_{n}\right\}$ of points in $\Pi X_{\nu}$ such that $z_{n+1} \in\left[V\left(n, J_{n}\right) \cap D\right] \backslash f^{-1}(x)$ where $J_{n}=J\left(z_{1}\right) \cup \cdots \cup J\left(z_{n}\right)$. It is now easy to see that the sequence $\left\{z_{n}\right\}$ converges to $y$ in $\Pi X_{\nu}$. Since each $z_{n}$ does not belong to $f^{-1}(x)$, it follows that $x=f(y)$ is a sequential limit point in $X$.

By mimicking the proof of Proposition 3.4 above one can prove the following: 
COROLLARY 3.5. Let $\left\{X_{\nu}: \nu \in I\right\}$ be a family of first countable spaces and let $Y$ be an arbitrary union of $\Sigma$-products of the $X_{\nu}$.

If $f$ is a continuous closed surjection from $Y$ onto a topological space $X$, then every nonisolated point of $X$ is a sequential limit point in $X$.

Since every dyadic space is the continuous image of a product of compact metric spaces, Proposition 3.4 generalizes [7, Corollary 9, p. 257] where it is shown that every nonisolated point in a dyadic space is a sequential limit point. We note that the conclusion of Proposition 3.4 is false if we only assume that every nonisolated point in each $X_{\nu}$ is a sequential limit point. For example let $B_{n}(n=1,2, \ldots)$ denote the Stone-Čech compactification of the integers and let $f: \Pi B_{n} \rightarrow B_{1}$ denote the projection onto the first coordinate. It is easy to see that every point in $\Pi B_{n}$ is a sequential limit point; however, by [10, Problem 60(6), p. 97] $B_{1}$ has no sequential limit points. We also note that the conclusion of Proposition 3.4 is false if $f$ is not closed. This follows from the fact that the one point compactification of an uncountable discrete space is the continuous image of a discrete space.

Following [18] we say that a Banach space $E$ is a Grothendieck space if each weak ${ }^{*}$ convergent sequence in the dual $E^{\prime}$ of $E$ is weakly convergent. By modifying the proof of $[12$, Théorème $9, \mathrm{p} .168]$ one can show that $C(X)$ is a Grothendieck space whenever $X$ is a basically disconnected compact space. The following proposition therefore generalizes [8, Theorem 12, p. 194] where it is shown that every dyadic subspace of a basically disconnected space is finite.

3.6. PROPOSITION. If $X$ is a completely regular pseudocompact space such that $C(X)$ is a Grothendieck space, then every subspace of $X$ having property $\mathrm{CM}$ is finite.

Proof. By [18, Remark 1, p. 192] it follows that $X$ has no infinite compact metric subspaces. To complete the proof it suffices to show that every infinite topological space having property $\mathrm{CM}$ has an infinite compact metric subspace. To see this let $Y$ be an infinite topological space having property $\mathrm{CM}$. By using [10, Theorem 0.13] it is easy to see that $C(Y)$ contains a function with infinite range, and, therefore $Y$ contains an infinite compact metric subspace.

\section{REFERENCES}

1. D. Amir and J. Lindenstrauss, The structure of weakly compactness in Banach spaces, Ann. of Math. (2) 88 (1968), 35-46. MR 37 \#4562.

2. G. Choquet, Lectures on analysis. Vol. I: Integration and topological vector spaces, Benjamin, New York, 1969. MR 40 \#3252; erratum, 44, p. 1630.

3. R. A. Conover, Normality and products of linearly ordered spaces, General Topology and Appl. 2 (1972), 215-225. MR 46 \#4499.

4. H. H. Corson, Normality in subsets of product spaces, Amer. J. Math. 81 (1959), 785-796. MR 21 \#5947. 
5. J. Diestel and R. H. Lohman, Applications of mapping theorems to Schwartz spaces and projections, Michigan Math. J. 20 (1973), 39-44. MR 47 \#5541.

6. J. Diestel, S. A. Morris and S. A. Saxon, Varieties of linear topological spaces, Trans. Amer. Math. Soc. 172 (1972), 207-230. MR 47 \#5540.

7. B. A. Efimov, Dyadic bicompacta, Trudy Moskov. Mat. Obß飞. 14 (1965), $211=$ Trans. Moscow Math. Soc. 14 (1965), 229-267. MR 34 \#1979.

8. B. A. Efimov and R. Engelking, Remarks on dyadic spaces. 11, Colloq. Math. 13 (1964/65), 181-197. MR 32 \#6391.

9. R. Engelking and A. Pelczynski, Remarks on dyadic spaces, Colloq. Math. 11 (1963), 55-63. MR 28 \#4504.

10. L. Gillman and M. Jerison, Rings of continuous functions, Univ. Ser. in Higher Math., Van Nostrand, Princeton, N.J., 1960. MR 22 \#6994.

11. S. Goldberg, On Sobczyk's projection theorem, Amer. Math. Monthly 76 (1969), 523-526. MR 39 \#6054.

12. A. Grothendieck, Sur les applications linéaires faiblement compactes d'espaces du type $C(K)$, Canad. J. Math. 5 (1953), 129-173. MR 15, 438.

13. R. C. James, Reflexivity and the sup of linear functionals, Israel J. Math. 13 (1972), 289-300 (1973). MR 49 \#3506.

14. G. Kothe, Über einen Satz von Sobczyk, An. Fac. Ci. Univ. Porto 49 (1966), 281-286. MR 37 \#3322.

15. J. Lindenstrauss, Weakly compact sets: Their topological properties and the Banach spaces they generate, Proc. Sympos. Infinite Dim. Topology (1967), Ann. of Math. Studies, no. 69, Princeton Univ. Press, Princeton, N.J., 1968, pp. 208-228.

16. A. Pelczynski, Linear extensions, linear averagings, and their applications to linear topological classification of spaces of continuous functions, Dissertationes Math. Rozprawy Math. 58 (1968). MR 37 \#3335.

17. - Projections in certain Banach spaces, Studia Math. 19 (1960), 209-228. MR 23 \#A3441.

18. H. P. Rosenthal, On quasi-complemented subspaces of Banach spaces, with an appendix on compactness of operators from $L^{P}(\mu)$ to $L^{r}(\nu)$, J. Functional Analysis 4 (1969), 176-214. MR 40 \#3277.

19. H. H. Schaefer, Topological vector spaces, Macmillan, New York, 1966. MR 33 \#1689.

20. A. Sobczyk, Projection of the space $(m)$ on its subspace $\left(c_{0}\right)$, Bull. Amer. Math. Soc. 47 (1941), 938-947. MR 3, 205.

21. W. A. Veech, Short proof of Sobczyk's theorem, Proc. Amer. Math. Soc. 28 (1971), 627-628. MR 43 \#879.

22. S. Willard, General topology, Addison-Wesley, Reading, Mass., 1970. MR 41 \#9173. GIA 30602

DEPARTMENT OF MATHEMATICS, UNIVERSITY OF GEORGIA, ATHENS, GEOR- 